\title{
Requerimientos germinativos de enredaderas características del Chaco Serrano de Córdoba, Argentina
}

\author{
Anahí Belén D’Agostino ${ }^{1}$, Diego Ezequiel Gurvich ${ }^{1,2}$, María Cecilia Ferrero", \\ Sebastián Rodolfo Zeballos ${ }^{1}$ \& Guillermo Funes ${ }^{1,2}$ \\ 1. Instituto Multidisciplinario de Biología Vegetal (CONICET-UNC), Av. Vélez Sársfield 299, CC 495, 5000, Córdoba, \\ Argentina; anahidagos@hotmail.com,mcferrero@conicet.gov.ar, sebazeba@hotmail.com \\ 2. Cátedra de Biogeografía, Departamento de Diversidad Biológica y Ecología, FCEFyN, Universidad Nacional de \\ Córdoba, Av. Vélez Sársfield 299, CC 495, 5000, Córdoba, Argentina; dgurvich@com.uncor.edu, \\ gfunes@imbiv.unc.edu.ar
}

Recibido 19-X-2011. Corregido 10-V-2012. Aceptado 06-VI-2012.

\begin{abstract}
Germinating requirements of common vines from Chaco Serrano of Córdoba, Argentina. Vines are conspicuous elements of floras in different ecosystems. Patterns of distribution and ecology of this group has been studied at regional scales, mainly in tropical areas, but less is known about factors affecting their distribution at smaller scales. In this study, the germinating requirements of common vines from two plant communities (open shrubland and woodland) in xerophytic mountain forests (Chaco serrano) were studied. A total of 21 species were selected and classified as typical of woodland, shrubland, or indifferent. Experimental treatments were three temperature regimes $\left(15-5^{\circ} \mathrm{C}, 25-15^{\circ} \mathrm{C}\right.$ and $\left.35-20^{\circ} \mathrm{C}\right)$, in light $(12-12 \mathrm{~h}$ daily photoperiod) and in continuous darkness. Total germination percentage, the relative light germination (RLG) and germination rate $\left(\mathrm{T}_{50}\right)$ were recorded. Among results we found that the temperature was the main factor triggering the germination process. No differences in the mean RLG and $\mathrm{T}_{50}$ between woodland and shrubland patches were found. The patterns observed in this work support the idea that, independently of patch type, germination is associated with temperature of the time of the year when most of the rainfall occurs. Germination of the studied species would be responding to macroclimate's factors (seasonality of climate and rainfall), rather than to a microclimate. This indicates that differences in species composition between patches would not be due to the factors studied, rather than to characteristics of the later stages of the cycle life. Rev. Biol. Trop. 60 (4): 15131523. Epub 2012 December 01.
\end{abstract}

Key words: Chaco forests, vines, seed germination, temperature, light.

Las plantas trepadoras, herbáceas y leñosas, son formas de vida que se caracterizan por estar enraizadas en el suelo (Ewers \& Fisher 1991), pero cuentan con otras plantas (principalmente árboles) $\mathrm{u}$ objetos externos para obtener soporte mecánico y una mayor exposición a la luz (Ewers \& Fisher 1991, Malizia \& Grau 2008). Los patrones de distribución de las enredaderas han sido estudiados en mayor medida en ambientes tropicales húmedos, siendo más escasa la información disponible para otros tipos de ecosistemas (Schnitzer \& Bongers 2002, Schnitzer 2005). A escala global, es conocido que la riqueza y abundancia de las mismas aumenta con la temperatura y la humedad (Gentry 1991, Rundel \& Franklin 1991, Teramura et al. 1991, Van Der Heijden \& Phillips 2009). Sin embargo, menos se conoce sobre los factores que afectan su distribución a escala local. En este sentido, algunos estudios indican que la disponibilidad de luz (Castellanos 1991, Teramura et al. 1991) y de soporte (Putz 1984) son los factores micro-ambientales más influyentes. En general, se ha asociado una mayor riqueza de trepadoras a sitios con algún grado de disturbio reciente (principalmente 
claros) (Schnitzer \& Carson 2001, Malizia \& Grau 2008).

Las diferentes actividades del hombre sobre los ecosistemas pueden ocasionar cambios en la estructura y composición de las comunidades vegetales que los componen $\mathrm{y}$, por consiguiente, en los componentes abióticos del sistema como disponibilidad de luz, agua o nutrientes (Breshears et al. 1997, Calder et al. 1993, 1997). La heterogeneidad ambiental, generada por las actividades mencionadas, puede ser percibida de diferentes maneras por las especies de plantas. El uso diferencial que éstas hacen sobre los distintos parches de hábitat puede deberse a diferencias en sus características regenerativas $\mathrm{y} / \mathrm{o}$ vegetativas. Particularmente, adquieren relevancia los caracteres de la fase regenerativa asociados a la semilla (tamaño, patrón de germinación y dormición), ya que el lugar y el momento de la germinación determinarán el ambiente en donde se desarrollará la plántula (Baskin \& Baskin 1998, Fenner \& Thompson 2005). En este sentido, el micrositio en el cual la semilla germina juega un rol importante en definir la distribución de la especie (Grubb 1977). Existe cierta relación entre los requerimientos ambientales para la germinación y las características ambientales predominantes en el hábitat, los que han sido interpretados como adaptaciones a condiciones ecológicas específicas (Grime et al. 1981, Mayer \& Poljakoff-Mayber 1989). Dentro de los factores abióticos que controlan la germinación, la luz (Baskin \& Baskin 1998, Pons 2000, Daws et al. 2002) y la temperatura (Baskin \& Baskin 1998, Probert 2000) aparecen como dos de los más relevantes (Funes et al. 2009).

Como producto de las actividades antrópicas, la vegetación de las partes más bajas de las Sierras de Córdoba se presenta como un mosaico de matorrales y bosques secundarios (Zak \& Cabido 2002). Ferrero (2010) estudió el patrón de distribución de enredaderas en el Bosque chaqueño serrano de Córdoba, donde constituyen un elemento importante de la flora nativa (Giorgis et al. 2011). Dicha autora encontró grupos de especies asociados a diferentes parches de vegetación, es decir, los bosques presentan una composición de especies distinta a la de los matorrales. Tal patrón podría estar asociado a las condiciones bióticas y abióticas diferenciales de cada sitio. En este sentido, los objetivos del presente trabajo fueron: 1) evaluar el efecto de la temperatura y la luz sobre la capacidad germinativa de enredaderas características de parches de bosques y matorrales; y 2) determinar si los requerimientos de germinación están relacionados con la fisonomía del paisaje.

Con base en lo argumentado hasta aquí, como hipótesis se postuló que habría diferencias en los requerimientos germinativos de las especies típicas de cada fisonomía (bosques y matorrales), en respuesta a diferentes condiciones ambientales. Particularmente, las especies típicas de bosques germinarían a temperaturas más bajas y serían indiferentes a la luz. Las especies típicas de matorrales se comportarían de manera contraria.

\section{MATERIALES Y MÉTODOS}

Área de estudio: El presente trabajo fue realizado en la Reserva Hídrica Natural Parque La Quebrada, en la ladera oriental de las Sierras Chicas de Córdoba, Argentina (3109'0" S - 64²0'00" W). Fitogeográficamente, se encuentra ubicada dentro del Distrito del Chaco serrano, en el piso del bosque serrano (Cabrera 1976, Luti et al. 1979). Los sitios de trabajo se ubicaron en ambos márgenes del lago La Quebrada (norte y sur), entre los 770-890msnm. En esta franja altitudinal, la vegetación se presenta como un mosaico de comunidades, entre las cuales se destacan matorrales y bosques (gran parte de ellos secundarios) (Zak \& Cabido 2002, Cabido et al. 2010). La zona presenta una temperatura media anual de $13^{\circ} \mathrm{C}$. Las precipitaciones están concentradas en primaveraverano, alcanzando los 700-800mm anuales (Vázquez et al. 1979). Los suelos son litosólicos de formación incipiente, perfil A-AC-C y con alto porcentaje de arenas y rocas de hasta $20 \mathrm{~cm}$ de diámetro, dentro del perfil y en superficie (Vázquez et al. 1979). 
En el área de estudio las formaciones vegetales presentan las características del matorral y bosque xerófilo que cohabita con pastizales. Las especies arbóreas dominantes de los bosques son Lithraea molleoides (Vell.) Engl. (Molle de beber), Zanthoxylum coco (Gillies) Engl. (Coco) y Celtis ehrenbergiana (Klotzsch) Liebm. (Tala). En el sotobosque se pueden encontrar especies como Ruprechtia apetala (Manzano del campo) y Bougainvillea stipitata Griseb (Falso tala). Por otro lado, los matorrales están dominados por Acacia caven (Molina) Molina (Espinillo), Condalia buxifolia (Piquillín) y Lantana camara L. (Bandera española). El estrato herbáceo está dominado por la gramínea en mata Jarava ichu Ruiz \& Pav, y por especies herbáceas como Bidens pilosa L. e Hyptis mutabilis (Rich.) Briq. (Luti et al. 1979, Giorgis et al. 2011).

Especies estudiadas y recolección de semillas: Se trabajó con 21 especies (16 géneros, 10 familias en total), las cuales fueron separadas en típicas de bosques, de matorrales e indiferentes (Ferrero 2010) (Cuadro 1). Asparagus setaceus y Vigna caracalla son exóticas para la flora del bosque serrano. La nomenclatura de las especies sigue a Instituto de Botánica Darwinion (Anónimo 1997).

La recolección se realizó durante febreroabril 2009, en nueve parches de bosque y cinco de matorral. Las semillas provinieron de al menos cinco individuos por especie, por sitio de estudio.

Las semillas fueron separadas de los frutos, limpiadas y colocadas en bolsas de papel, en las que permanecieron almacenadas a temperatura ambiente y en oscuridad, hasta el momento de realizar los experimentos.

Medición de luz: Con el fin de caracterizar la luz que llega al suelo en cada fisonomía considerada (bosques y matorrales), se registró la calidad (a través de la relación rojo/rojo lejano; R/RL, 660:730nm) y la cantidad de luz (densidad de radiación fotosintéticamente activa; RFA, $\mu \mathrm{mol} / \mathrm{m}^{2}$.s) en cada tipo de parche estudiado. Para la primera, se utilizó un sensor
Skye (SKR 110) y para la segunda, un sensor LICOR. Las lecturas fueron tomadas en tres sitios por fisonomía; en total se obtuvieron 225 lecturas para R/RL y 225 para RFA, para cada tipo de parche. Luego se calcularon los promedios de cada grupo. Las mediciones fueron realizadas durante junio-julio del 2010, en días despejados, entre las 12 y $15 \mathrm{hr}$. Dicho horario fue elegido de manera arbitraria, con el fin de unificar el momento del día para todas las mediciones.

Experimento de germinación: Se tuvieron en cuenta tres repeticiones por tratamiento, con 20 semillas cada una. Éstas fueron dispuestas en cápsulas de Petri de nueve centímetros de diámetro sobre papel de filtro, regadas con agua destilada y colocadas en cámaras de germinación en condiciones controladas de temperatura y luz. Las semillas fueron expuestas a tres regímenes de temperatura: $15-5^{\circ} \mathrm{C}$, $25-15^{\circ} \mathrm{C}$ y $35-20^{\circ} \mathrm{C}$, y dos condiciones de luz: un fotoperíodo de 12-12hr (luz-oscuridad) y oscuridad permanente. Para esta última condición las cápsulas fueron cubiertas con doble capa de papel de aluminio. Las temperaturas se seleccionaron teniendo en cuenta las medias máximas y mínimas de las estaciones de invierno, primavera/otoño y verano en el sitio de estudio (Capitanelli 1979). La luz fue provista por tubos fluorescentes de luz fría (400-700nm), con una densidad de $\sim 38 \mu \mathrm{mol} / \mathrm{m}^{2}$.s. Todos los experimentos tuvieron una duración de 31 días.

En cada tratamiento se evaluó el porcentaje final de germinación (Cuadro 1). El criterio para considerar una semilla como germinada fue la emergencia en dos milímetros de la radícula (ISTA 1996). En las cápsulas en condiciones de luz, el registro de las germinaciones y el riego se realizó diariamente, mientras que en las cápsulas sometidas a los tratamientos de oscuridad, la germinación fue controlada al final de cada experimento. No obstante, fueron regadas una vez por semana, en horas de baja calidad de luz, realizando una mínima apertura del sobre de aluminio.

Cabe aclarar que las semillas de aquellas especies en las que se conoce una dormición 


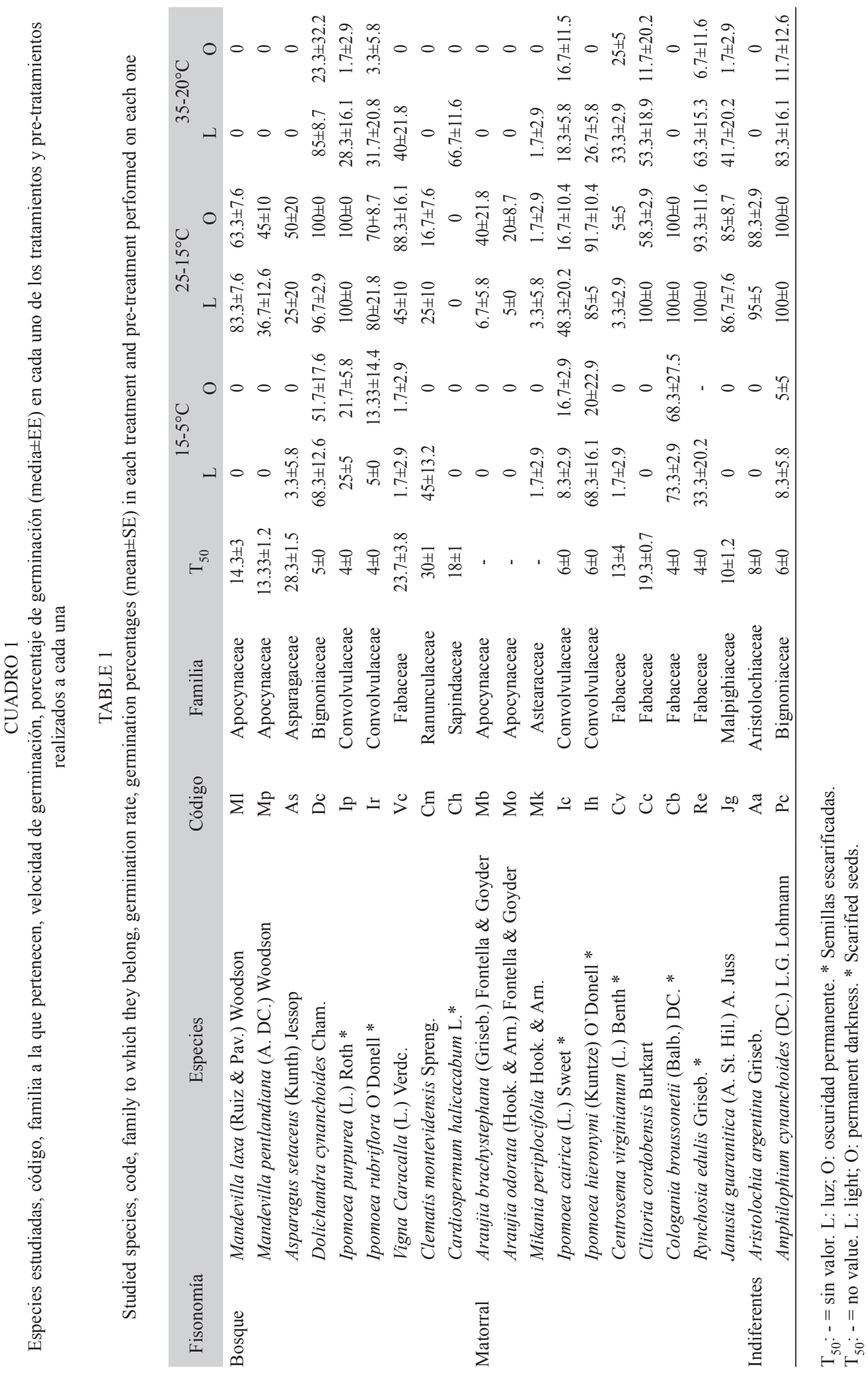


física fueron sometidas a un pre-tratamiento, que consistió en una escarificación con papel de lija, para asegurar la imbibición de las mismas (Baskin \& Baskin 1998) (Cuadro 1).

Para verificar la respuesta de las semillas a la luz se utilizó el índice de germinación relativa a la luz (GRL), el cual expresa los requerimientos de luz para la germinación (Milberg et al. 2000).

$$
\mathrm{GRL}=\mathrm{GL} /(\mathrm{GO}+\mathrm{GL})
$$

En donde $\mathrm{GL}=\%$ germinación a la luz, y $\mathrm{GO}=\%$ germinación en oscuridad permanente.

Este índice fue calculado sólo para aquellas especies que presentaron un porcentaje de germinación igual o mayor al $50 \%$, en el rango de temperatura óptimo (aquel en el que la especie registró el mayor porcentaje de germinación en luz u oscuridad), debido a que una germinación menor podría indicar algún mecanismo de dormición involucrado (Milberg et al. 2000, Flores et al. 2006) (Cuadro 1).

El GRL representa un rango de valores que van desde cero (germinación sólo en oscuridad) a uno (germinación sólo en luz). Las especies con GRL superior a 0.75 se consideraron dependientes de la luz (fotoblásticas positivas), mientras que aquéllas con índice menor a 0.25 fueron consideradas repelentes de la luz (fotoblásticas negativas). Las especies con valores entre 0.25-0.75 se clasificaron como indiferentes a la luz.

Por otra parte, se obtuvo la velocidad de germinación $\left(\mathrm{T}_{50}\right)$, medida como el número de días para alcanzar el 50\% de la germinación observada (en la temperatura óptima y bajo condiciones de 12-12hr luz-oscuridad). El $\mathrm{T}_{50}$ fue calculado sólo para especies con una germinación igual o mayor al 20\% (Van Assche \& Vandelook 2006).

Los análisis se realizaron utilizando el paquete estadístico InfoStat (Di Rienzo et al. 1999). Éstos se basaron en dos grupos de especies: típicas o preferentes de bosques y típicas o preferentes de matorrales. En primera instancia se consideró un tercer grupo de especies indiferentes (presentes en ambas fisonomías por igual), no obstante, al estar conformado sólo por dos especies no fue incluido en los análisis (Ferrero 2010) (Cuadro 1).

Con el fin de determinar si existen diferencias significativas en la calidad $(\mathrm{R} / \mathrm{RL})$ y la cantidad de luz (RFA) que llega al suelo, en los dos tipos de parches estudiados, se realizó la prueba de Wilcoxon $(\mathrm{p}<0.05)$ (Sokal \& Rohlf 1995), debido a la falta normalidad de los datos.

Los resultados correspondientes a los ensayos de germinación, para el total de especies típicas de cada fisonomía, se analizaron mediante Análisis de la varianza (Anova) con rangos (no paramétrico), puesto que la variable porcentaje de germinación, en cada situación experimental, no cumplió con los supuestos de normalidad ni homogeneidad de varianzas (Conover \& Iman 1981, Hora \& Conover 1984, Zar 1999, Urcelay et al. 2003), aun cuando los porcentajes fueron transformados en $\sqrt{ }$ arcoseno. Este Anova con rangos fue tri-factorial, considerando a la fisonomía, la temperatura y la luz/oscuridad como los tres factores principales. Se aplicó un LDS de Fischer como test a posteriori. La interpretación de los resultados que arroja este tipo de análisis, se realiza de la misma manera que para un Anova paramétrico (Zar 1999, Urcelay et al. 2003).

Las diferencias entre bosques y matorrales con respecto al GRL promedio fueron evaluadas mediante la prueba de Wilcoxon $(\mathrm{p}<0.05)$ (Sokal \& Rohlf 1995).

Por otro lado, para el $\mathrm{T}_{50}$ se utilizó la prueba $\mathrm{T}$ de comparación de medias $(\mathrm{p}<0.05)$ (Sokal \& Rohlf 1995), luego de transformar los datos en $\log _{10}$.

\section{RESULTADOS}

Se registraron diferencias significativas en la relación $\mathrm{R} / \mathrm{RL}$ en los dos tipos de parches estudiados $(\mathrm{w}=2$ 755.5, $\mathrm{p}=0.0001)$. El valor obtenido para bosques fue de $0.66 \pm 0.03 \mathrm{~nm}$, mientras que para matorrales fue de $0.89 \pm 0.01 \mathrm{~nm}$. Por otro lado, la RFA también resultó estadísticamente diferente para ambos sitios $(\mathrm{w}=1225$, $\mathrm{p}=0.0001)$. Se registraron $88.77 \pm 18.8 \mu \mathrm{mol} /$ 
$\mathrm{m}^{2}$.s en bosques, y $477.94 \pm 43.42 \mu \mathrm{mol} / \mathrm{m}^{2}$.s en los sitios abiertos. En ambos casos, la calidad y cantidad de luz respectivamente, fueron significativamente mayores en los matorrales.

La germinación no fue afectada de manera significativa por la fisonomía $(\mathrm{F}=0.02, \mathrm{p}=0.8902)$. No obstante, la temperatura $(\mathrm{F}=23.97, \mathrm{p}=0.0001)$ y la luz $(\mathrm{F}=4.26$, $\mathrm{p}=0.0416)$ sí influyeron significativamente en dicho proceso. De estos dos factores, la temperatura fue el más importante. No hubo interacción entre ninguno de los tratamientos (temperatura $\mathrm{x}$ luz $\mathrm{p}=0.2102$; fisonomía $\mathrm{x}$ luz $\mathrm{p}=0.9757$; temperatura $\mathrm{x}$ fisonomía $\mathrm{p}=0.8585$; temperatura $\mathrm{x}$ fisonomía $\mathrm{x}$ luz $\mathrm{p}=0.9196$ ) (Fig. 1).

De los tres regímenes considerados, todas las especies germinaron en al menos un tratamiento. El porcentaje de germinación varió entre $1.7 \%-100 \%$ (Cuadro 1). La temperatura óptima de germinación para el total de especies estudiadas fue $15-25^{\circ} \mathrm{C}$, tanto en condiciones de luz como en oscuridad permanente. No hubo diferencias entre las dos condiciones restantes, las cuales registraron porcentajes de germinación significativamente menores al obtenido en la temperatura óptima (Cuadro 1 y Fig. 1). La especie que más germinó (independientemente de la temperatura óptima) fue Dolichandra cynanchoides, seguida por Ipomoea purpurea. Éstas germinaron en todos los tratamientos a los que fueron sometidas (Cuadro 1). El resto de las especies del género Ipomoea y Amphilophium cynanchoides mostraron altos porcentajes de germinación y requerimientos poco estrictos, con respecto al resto de las especies estudiadas (Cuadro 1). Por otra parte, Araujia brachystephana y Araujia odorata fueron las que menos germinaron, y junto con Cardiospermum halicacabum, mostraron condiciones muy estrictas para germinar. Si bien C. halicacabum germinó bien (más del 50\%), lo hizo en una única condición $\left(20-35^{\circ} \mathrm{C}\right.$, luz) (Cuadro 1$)$.

Con respecto al GRL, 11 de las 12 especies para las cuales se calculó este índice, resultaron indiferentes a la luz (A. setaceus, V. caracalla, D. cynanchoides, I. purpurea, I. rubriflora, Mandevilla laxa, I. hieronymi, Cologania broussonetii, Janusia guaranitica, Rynchosia edulis y Clitoria cordobensis). Sólo C. halicacabum resultó fotoblástica positiva (Fig. 2). En cuanto al GRL promedio para cada fisonomía

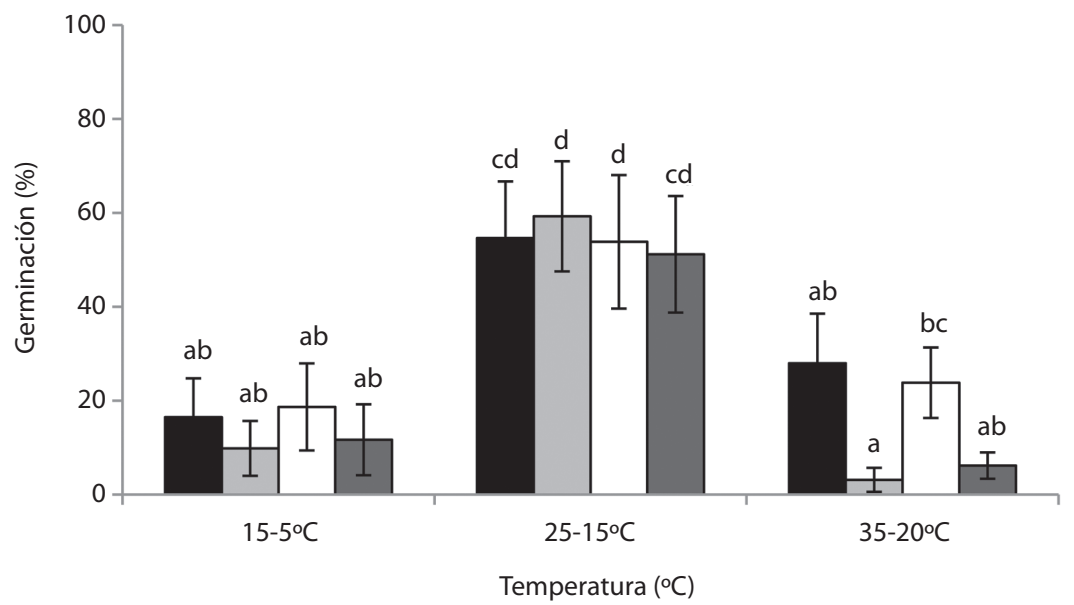

Fig. 1. Germinación (media \pm ES) de las especies típicas de cada físonomía. Barras negras: luz, bosque; barras gris claro: oscuridad, bosque; barras blancas: luz, matorral; barras gris oscuro: oscuridad, matorral. Diferentes letras indican diferencias significativas (prueba LSD de Fisher, $\mathrm{p}<0.05$ ).

Fig. 1. Germination (mean \pm SE) of the typical species of each site. Black bars: light, woodland; light grey bars: darkness, woodland; white bars: light, shrubland; dark grey bars: darkness, shrubland. Different letters indicate significant differences (test LSD, $\mathrm{p}<0.05$ ). 


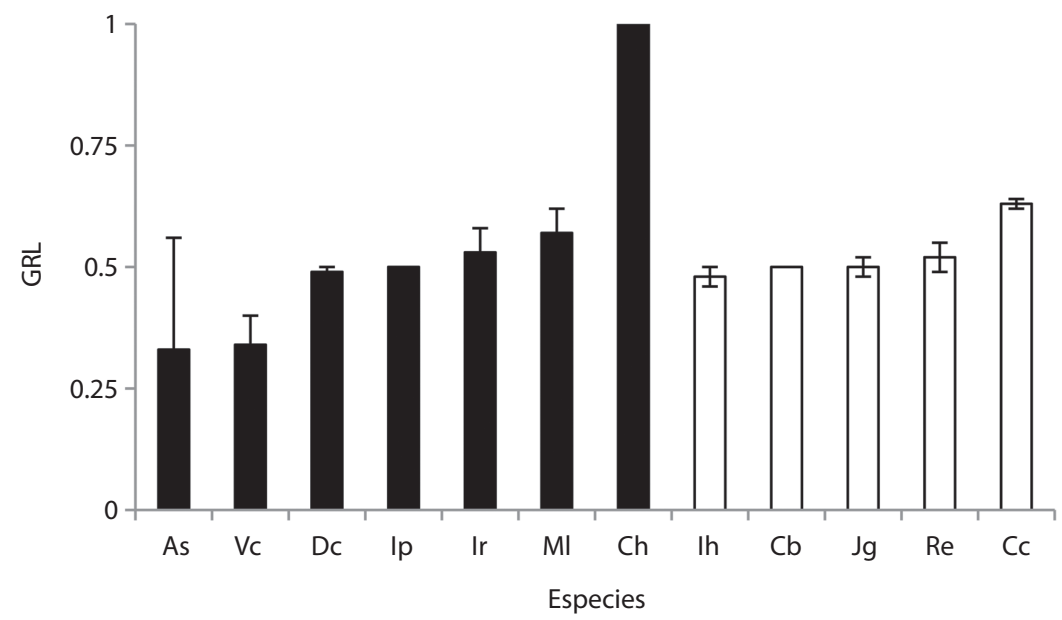

Fig. 2. Germinación relativa a la luz (GRL). Barras negras: especies típicas de bosques; barras blancas: especies típicas de matorrales. Los códigos se detallan en el cuadro 1.

Fig. 2. Relative light germination (RLG). Black bars: woodlands species; white bars: shrublands species. The codes are listed in table 1.

$(0.54 \pm 0.08$ para bosques y $0.53 \pm 0.03$ para matorrales), no se observaron diferencias significativas $(\mathrm{w}=34, \mathrm{p}=0.876)$.

La velocidad de germinación osciló entre 4 y 30 días (Cuadro 1). Representantes de las familias Convolvulaceae (I. purpurea e $I$. rubriflora) y Fabaceae (C. broussonetii y $R$. edulis) fueron las que germinaron más rápido, mientras que Clematis montevidensis y $A$. setaceus fueron las que más tardaron en alcanzar el $50 \%$ de la germinación total (Cuadro 1). El valor de $\mathrm{T}_{50}$ promedio registrado para bosques $(15.6 \pm 10.2)$ y matorrales $(8.9 \pm 5.7)$ no fue estadísticamente diferente $(\mathrm{t}=1.56, \mathrm{p}=0.14)$.

\section{DISCUSIÓN}

Los resultados obtenidos no apoyan la hipótesis planteada, ya que las especies típicas de bosques y matorrales mostraron los mismos requerimientos germinativos con respecto a las distintas condiciones de temperatura y luz a las que fueron sometidas. De estos dos factores, la temperatura fue el principal regulador del proceso de germinación. Para el total de especies estudiadas, el rango de temperatura óptimo para la germinación coincide con las temperaturas estivales, momento en el cual se producen las precipitaciones en el área de estudio. Patrones similares han sido reportados por Funes \& Venier (2006), Gurvich et al. (2008), Funes et al. (2009) y Zalazar et al. (2009), para distintas especies de la región chaqueña argentina. También se ha encontrado este patrón para especies de bosques y matorrales mediterráneos (Bell et al. 1993, Bell 1994, 1999, Figueroa et al. 1996, Turner et al. 2005). Este comportamiento aseguraría que la máxima germinación se produzca en el momento del año en el que las probabilidades de supervivencia de las plántulas son mayores (Baskin \& Baskin 1998, Turner et al. 2005).

La luz no tuvo una influencia importante en el proceso de germinación de las especies estudiadas. Resultados similares han sido reportados por Funes et al. (2009) para especies de distintas formas de vida del Chaco occidental de Argentina. Posiblemente la luz no sea determinante para la germinación en los sistemas chaqueños, sino que otros factores ambientales estarían regulando este proceso (como la estacionalidad térmica, asociada a la variación estacional en las precipitaciones). Es probable que este factor sea relevante sólo para 
C. halicacabum, que resultó la única especie fotoblástica positiva. Es remarcable el hecho de que la única especie dependiente de la luz sea típica de bosques, lo cual no se corresponde con la hipótesis planteada. Otros autores han encontrado una germinación nula de $C$. halicacabum en diferentes condiciones (Jurado et al. 2000). Ante ésto, resulta difícil establecer un comportamiento preciso en esta especie.

Varias de las especies estudiadas (Mandevilla pentlandiana, C. montevidensis, A. odorata, A. brachystephana y Mikania periplocifolia), germinaron en bajos porcentajes (no superaron el 50\%) y, en general, en un estrecho rango de condiciones, lo que sugiere la presencia de algún tipo de dormición en ellas. Si bien hay poca información disponible acerca de los patrones de germinación y dormición de las semillas de especies trepadoras, los datos indicarían la presencia de dormición morfológica o morfo-fisiológica (Williams \& Buxton 1995, Baskin \& Baskin 1998). Por otro lado, aquellas especies a las cuales se les realizó una escarificación mecánica, por poseer cubiertas duras e impermeables al agua (dormición física sensu; Baskin \& Baskin 1998), como I. purpurea, I. rubriflora, I. cairica, I. hieronymi, Centrosema virginianum, C. broussonetii y R. edulis, germinaron en un rango amplio de condiciones. Esto coincide con lo expresado por Baskin \& Baskin (1998) y Baskin et al. $(2000,2004)$ en relación a que las semillas que poseen dormición física germinan en amplias condiciones de luz y temperatura una vez que son escarificadas natural $\mathrm{o}$ artificialmente.

Con respecto a la velocidad de germinación, no se observaron diferencias significativas entre las especies de ambas fisonomías. Podría haberse esperado un $\mathrm{T}_{50}$ menor en las especies de bosque, teniendo en cuenta que estos parches (debido a la menor calidad y cantidad de luz que llega al suelo), podrían considerarse micrositios estresantes para la germinación (Leishman et al. 2000). Ante esto, cabría esperar que dichas especies sean menos estrictas en sus requerimientos germinativos, lo que implicaría, entre otras cosas, indiferencia a la luz y mayor velocidad de germinación
(Pearson et al. 2003). El hecho de no encontrar diferencias en este sentido (y además en el GRL promedio para cada fisonomía), posiblemente se deba, como ya se mencionó, a que la luz no actúa como un factor limitante en los sistemas chaqueños, y por lo tanto el comportamiento germinativo de las semillas estudiadas es independiente de la misma.

Teniendo en cuenta el trabajo realizado por Ferrero (2010), las especies estudiadas no responden de la misma manera durante las fases regenerativa y vegetativa adulta, a los factores ambientales particulares de cada fisonomía. Dicha autora encontró una composición diferencial de especies entre un tipo de parche y otro, y una mayor diversidad de estrategias funcionales (relacionadas a la velocidad de crecimiento, forrajeo de luz y uso del agua) en los parches de bosque respecto al matorral. No obstante, el presente trabajo muestra una respuesta regenerativa prácticamente igual por parte de las especies típicas de cada fisonomía a las condiciones bióticas y abióticas particulares de cada comunidad vegetal. Esto concuerda con lo expuesto por Díaz \& Cabido (1997) y Díaz et al. (1998), quienes postulan que no necesariamente existe una relación o condicionamiento entre las características reproductivas $\mathrm{y}$ vegetativas.

Los patrones observados en este estudio apoyan la idea de que la germinación se asocia con las temperaturas del momento del año en el que se concentran las precipitaciones, y por lo tanto, las probabilidades de supervivencia de las plántulas son mayores. La germinación de las especies estudiadas estaría respondiendo principalmente a factores macro-climáticos (estacionalidad del clima y de las precipitaciones), más que a factores micro-climáticos (parches con distinta fisonomía), siendo éste un comportamiento de valor adaptativo, por parte de las especies, al clima regional (Baskin \& Baskin 1998, Turner et al. 2005). Dicho comportamiento posiblemente ha maximizado el éxito de las especies chaqueñas a lo largo de la evolución, en sistemas con estacionalidad térmica e hídrica marcada y canopias relativamente abiertas. 
Finalmente, los resultados encontrados sugieren que no existirían diferencias en algunas de las características de la fase regenerativa de las enredaderas estudiadas. Esto indica que las diferencias en la composición de especies entre los parches, no sería debido a los factores aquí estudiados, sino más bien a características de las etapas posteriores del ciclo de vida (Ferrero 2010).

\section{AGRADECIMIENTOS}

A CONICET (PIP 1286) y FONCyT (PICT 2006, N ${ }^{\circ}$ 903) por la financiación del presente trabajo. A Paula Venier por el apoyo brindado.

\section{RESUMEN}

Las enredaderas son elementos conspicuos de la flora de diferentes ecosistemas. Numerosos estudios basados en patrones de distribución y ecología de este grupo de plantas se han realizado a escala regional, se conoce poco sobre los factores que afectan su distribución a nivel local. Se estudiaron los requerimientos germinativos de 21 enredaderas del Bosque chaqueño serrano de Córdoba, las cuales fueron clasificadas en típicas de bosques, matorrales e indiferentes. Se llevaron a cabo experimentos de germinación en tres regímenes de temperatura $\left(15-5^{\circ} \mathrm{C}, 25-15^{\circ} \mathrm{C}\right.$ y $\left.35-20^{\circ} \mathrm{C}\right)$, en luz $(12-12 \mathrm{hr}$ luz-oscuridad) y oscuridad permanente. Se registró el porcentaje final de germinación, el índice de germinación relativa a la luz (GRL) y la velocidad de germinación $\left(\mathrm{T}_{50}\right)$. La temperatura fue el principal factor regulador del proceso germinativo. El GRL y el $\mathrm{T}_{50}$ no registraron diferencias entre fisonomías. Los patrones observados apoyan la idea de que la germinación de este grupo de plantas estaría respondiendo a factores macroclimáticos (estacionalidad del clima y las precipitaciones), más que a un microclima (factores asociados a la fisonomía del sitio). La composición diferencial de enredaderas en los parches de bosques y arbustos no estaría explicada por los factores estudiados, sino por características de las etapas posteriores a la semilla.

Palabras clave: Chaco serrano, características regenerativas, enredaderas, semillas, temperatura, luz.

\section{REFERENCIAS}

Anónimo. 1997. Flora del Conosur, Catálogo de plantas vasculares. Instituto de botánica Darwinion, Academia Nacional de Ciencias Exactas, Físicas y Naturales, CONICET, San Isidro, Buenos Aires, Argentina. (Consultado: Febrero 20, 2012, www.darwin.edu.ar).
Baskin, J.M., B. Davis, C.C. Baskin, S. Gleason \& S. Cordell. 2004. Physical dormancy in seeds of Dodonea viscosa (Sapindales, Sapindaceae) from Hawaii. Seed Sci. Res. 14: 81-90.

Baskin, J.M., C.C. Baskin \& X. Li. 2000. Taxonomy, anatomy and evolution of physical dormancy in seeds. Plant Spec. Biol. 15: 139-152.

Baskin, C.C. \& J.M. Baskin. 1998. Seed. Ecology, biogeography and evolution of dormancy and germination. Academic, San Diego, California, EEUU.

Bell, D. 1994. Interaction of fire, temperature and light in the germination response of 16 species from the Eucalyptus marginata forest of south-western Western Australia. Aust. J. Bot. 42: 501-509.

Bell, D. 1999. The process of germination in Australian species. Aust. J. Bot. 47: 475-517.

Bell, D., J. Plummer \& S. Taylor. 1993. Seed germination ecology in southwestern Western Australia. Bot. Rev. 76: 24-73.

Breshears, D.D., P.M. Rich, F.J. Barnes \& K. Campbell. 1997. Overstory-imposed heterogeneity in solar radiation and soil moisture in a semiarid woodland. Ecol. Appl. 7: 1201-1215.

Cabido, M., M. Giorgis \& M. Tourn. 2010. Guía para una excursión botánica en las Sierras de Córdoba. Bol. Soc. Argent. Bot. 45: 209-219.

Cabrera, A.L. 1976. Regiones fitogeográficas argentinas. Enciclopedia Argentina de Agricultura y Jardinería. Tomo II. Acme, Buenos Aires, Argentina.

Calder, I.R., M.J. Hall \& K.T. Prasanna. 1993. Hydrological impact of Eucalyptus plantation in India. J. Hydrol. 150: 635-648.

Calder, I.R., P.T.W. Rosier, K.T. Prasanna \& S. Parameswarappa. 1997. Eucalyptus water use greater than rainfall input a possible explanation from southern India. Hydrol. Earth. Syst. Sci. 1: 249-256.

Capitanelli, R. 1979. Clima, p. 45-138. In J. Vázquez, R. Miatello \& M. Roquén (eds.). Geografía física de la provincia de Córdoba. Boldt, Buenos Aires, Argentina.

Castellanos, A.E. 1991. Photosynthesis and gas exchange of vines, p. 181-204. In F.E. Putz \& H.A. Mooney (eds.). The biology of vines. Cambridge University, Cambridge, Reino Unido.

Conover, W.J. \& R.L. Iman. 1981. Rank transformations as a bridge between parametric and nonparametric statistics. J. Am. Stat. Assoc. 35: 124-129.

Daws, M.I., D.F.R.P. Burslem, L.M. Crabtree, P. Kirkman, C.E. Mullins \& J.W. Dalling. 2002. Differences in seed germination responses may promote coexistence of four sympatric Piper species. Funct. Ecol. 16: 258-267. 
Di Rienzo, J., W. Bobledo, F. Casanoves, M. Balzarini, L. González, C.W. Robledo \& E.M. Tablada. 1999. InfoStat, Estadística y Biometría. Facultad de Ciencias Agropecuarias, Universidad Nacional de Córdoba, Córdoba, Córdoba, Argentina.

Díaz, S. \& M. Cabido. 1997. Plant functional types and ecosystem function in relation to global change. J. Veg. Sci. 8: 463-474.

Díaz, S., M. Cabido \& F. Casanoves. 1998. Plant functional traits and environmental filters at a regional scale. J. Veg. Sci. 9: 113-122.

Ewers, F.W. \& J.B. Fisher. 1991. Why vines have narrow stems: histological trends in Bauhinia (Fabaceae). Oecologia 88: 233-237.

Fenner, M. \& K. Thompson. 2005. The ecology of seeds. Cambridge University, Cambridge, Reino Unido.

Ferrero, M.C. 2010. Distribución y características funcionales de enredaderas de distintas comunidades vegetales del Chaco Serrano (Reserva La Quebrada, Córdoba). Tesina de graduación, Universidad Nacional de Córdoba, Córdoba, Argentina.

Figueroa, J., J. Armesto \& J.F. Hernández. 1996. Estrategias de germinación y latencia de semillas en especies del bosque templado de Chiloé, Chile. Rev. Chil. Hist. Nat. 69: 260-299.

Flores, J., E. Jurado \& A. Arredondo. 2006. Effect of light on germination of seeds of Cactaceae from the Chihuahuan Desert, México. Seed Sci. Res. 16: 149-155.

Funes, G. \& P. Venier. 2006. Dormancy and germination in three Acacia (Fabaceae) species from central Argentina. Seed Sci. Res. 16: 77-82.

Funes, G., S. Díaz \& P. Venier. 2009. La temperatura como principal determinante de la germinación en especies del Chaco seco de Argentina. Ecol. Austral. 19: 129-138.

Gentry, A.H. 1991. The distribution and evolution of climbing plants, p. 3-49. In F.E. Putz \& H.A. Mooney (eds.). The biology of vines. Cambridge University, Cambridge, Reino Unido.

Giorgis, M.A., A.M. Cingolani, F. Chiarini, J. Chiapella, G. Barboza, L. Ariza Espinar, R. Morero, D.E. Gurvich, P.A. Tecco, R. Subils \& M. Cabido. 2011. Composición florística del Bosque chaqueño serrano en la provincia de Córdoba, Argentina. Kurtziana 36: 9-43.

Grime, J.P., G. Mason, A.V. Curtis, J. Rodman, S.R. Band, M.A.G. Mowforth, A.M. Neal \& S. Shaw. 1981. A comparative study of germination characteristics in a local flora. J. Ecol. 69: 1017-1059.

Grubb, P.J. 1977. The maintenance of species richness in plant communities: the importance of the regeneration niche. Biol. Rev. 52: 107-145.

Gurvich, D., G. Funes, M. Giorgis \& P. Demaio. 2008. Germination characteristics of four coexisting
Gymnocalycium (Cactaceae) species with different flowering phenologies. Nat. Area. J. 28: 104-108.

Hora, S.C. \& W.J. Conover. 1984. The $F$ statistic in the two-way layout with rank-score transformed data. J. Am. Stat. Assoc. 79: 668-673.

ISTA (International Seed Testing Association). 1996. International rules for seed testing. Rules Zurich, Zurich, Suiza.

Jurado, E., O. Aguirre, J. Flores, J. Navar, H. Villalón \& D. Wester. 2000. Germination in tamaulipan thornscrub of northeastern México. J. Arid Environ. 46: 413-424.

Leishman, M.R., I.J. Wright, A.T. Moles \& M. Westoby. 2000. The evolutionary ecology of seed size, p. 31-51. In M. Fenner (ed.). Seeds: the ecology of regeneration in plant communities. CAB International, Wallingford, Reino Unido.

Luti, R., M. Solís, M. Galera, N. Müller, M. Berzal, M. Nores, M. Herrera \& J. Barrera. 1979. Vegetación, p. 297-368. In J. Vázquez, R. Miatello \& M. Roque (eds.). Geografía física de la provincia de Córdoba. Boldt, Buenos Aires, Argentina.

Malizia, A. \& H.R. Grau. 2008. Landscape context and microenvironment influences on liana communities within treefall gaps. J. Veg. Sci. 19: 597-604.

Mayer, A.M. \& A. Poljakoff-Mayber. 1989. The germination of seeds. Pergamon, Oxford, EEUU.

Milberg, P., L. Andersson \& K. Thompson. 2000. Largeseeded species are less dependent on light for germination than small-seeded ones. Seed Sci. Res. 10: 99-104.

Pearson, T.R.H., D.F.R.P. Burslem, C.E. Mullins \& J.W. Dalling. 2003. Functional significance of photoblastic germination in neotropical pioneer trees: a seed's eye view. Funct. Ecol. 17: 394-402.

Pons, T.L. 2000. Seed responses to light, p. 237-260. In M. Fenner (ed.). Seeds: the ecology of regeneration in plant communities. CAB International, Wallingford, Reino Unido.

Probert, R.J. 2000. The role of temperature in the regulation of seed dormancy and germination, p. 261-292. In M. Fenner (ed.). Seeds: the ecology of regeneration in plant communities. CAB International, Wallingford, Reino Unido.

Putz, F.E. 1984. The natural history of lianas on Barro Colorado Island, Panama. Ecology 65: 1713-1724.

Rundel, P.W. \& T. Franklin. 1991. Vines in arid and semiarid ecosystems, p. 337-356. In F.E. Putz \& H.A. Mooney. The biology of vines. Cambridge University, Cambridge, Reino Unido.

Schnitzer, S.A. 2005. A mechanistic explanation for global patterns of liana abundance and distribution. Am. Nat. 166: 262-276. 
Schnitzer, S.A. \& W.P Carson. 2001. Treefall gaps and the maintenance of species diversity in a tropical forest. Ecology 82: 913-919.

Schnitzer, S.A. \& F. Bongers. 2002. The ecology of lianas and their role in forests. Trends. Ecol. Evol. 17: 223-230.

Sokal, R. \& F. Rohlf. 1995. Biometry. Freeman, Nueva York, EEUU.

Teramura, A.H., W.G. Gold \& I.N. Forseth. 1991. Physiological ecology of mesic, temperate woody lianas, p. 245-285. In F.E. Putz \& H.A. Mooney (eds.). The biology of vines. Cambridge University, Cambridge, Reino Unido.

Turner, S.R., D. Merritt, C.C. Baskin, K. Dixon \& J.M. Baskin. 2005. Physical dormancy in seeds of six genera of Australian Rhamnaceae. Seed Sci. Res. 15: $51-58$.

Urcelay, C., M.S. Bret-Harte, S. Díaz \& F.S. Chapin. 2003. Mycorrhizal colonization mediated by species interactions in arctic tundra. Oecologia 137: 399-404.

Van Assche, J.A. \& F.E.A. Vandelook. 2006. Germination ecology of eleven species of Geraniaceae and
Malvaceae, with special reference to the effects of drying seeds. Seed Sci. Res. 16: 283-290.

Van Der Heijden, G.M.F. \& O.L. Phillips. 2009. Environmental effects on Neotropical liana species richness. J. Biogeogr. 36: 1561-1572.

Vázquez, J.B., R.A. Miatello \& M. Roque. 1979. Geografía Física de la Provincia de Córdoba. Banco Provincia de Córdoba, Córdoba, Argentina.

Williams, P. \& R. Buxton. 1995. Aspects of the ecology of two species of Passiflora (P. mollissima (Kunth) L. Bailey and $P$. pinnatistipula (Cav.) as weeds in South Island, New Zealand. New Zeal. J. Bot. 33: 315-323.

Zak, M.R. \& M. Cabido. 2002. Spatial patterns of the Chaco vegetation of central Argentina: integration of remote sensing and phytosociology. Appl. Veg. Sci. 5: 213-226.

Zalazar, M., G. Funes \& P. Venier. 2009. Factores que afectan la germinación de Justicia squarrosa Griseb., forrajera nativa de la región chaqueña de la Argentina. Agriscientia 26: 1-6.

Zar, J.H. 1999. Biostatistical analysis. Prentice Hall, Nueva Jersey, EEUU. 
